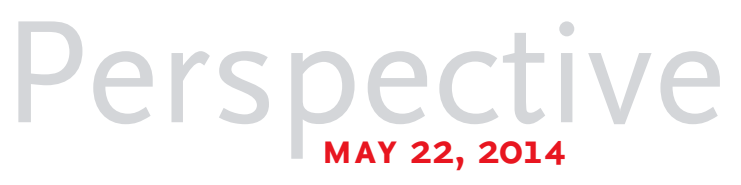

\title{
Abolishing Mammography Screening Programs? A View from the Swiss Medical Board
}

Nikola Biller-Andorno, M.D., Ph.D., and Peter Jüni, M.D.

\section{Tn January 2013, the Swiss Medical Board, an in- dependent health technology assessment initiative under the auspices of the Conference of Health Ministers of the Swiss Cantons, the Swiss Medical}

Association, and the Swiss Academy of Medical Sciences, was mandated to prepare a review of mammography screening. The two of us, a medical ethicist and a clinical epidemiologist, were members of the expert panel that appraised the evidence and its implications. The other members were a clinical pharmacologist, an oncologic surgeon, a nurse scientist, a lawyer, and a health economist. As we embarked on the project, we were aware of the controversies that have surrounded mammography screening for the past 10 to 15 years. When we reviewed the available evidence and contemplated its implications in detail, however, we became increasingly concerned.

First, we noticed that the ongo- ing debate was based on a series of reanalyses of the same, predominantly outdated trials. The first trial started more than 50 years ago in New York City and the last trial in 1991 in the United Kingdom. ${ }^{1}$ None of these trials were initiated in the era of modern breast-cancer treatment, which has dramatically improved the prognosis of women with breast cancer. Could the modest benefit of mammography screening in terms of breast-cancer mortality that was shown in trials initiated between 1963 and 1991 still be detected in a trial conducted today?

Second, we were struck by how nonobvious it was that the benefits of mammography screening outweighed the harms. The relative risk reduction of approximate- ly $20 \%$ in breast-cancer mortality associated with mammography that is currently described by most expert panels ${ }^{2}$ came at the price of a considerable diagnostic cascade, with repeat mammography, subsequent biopsies, and overdiagnosis of breast cancers - cancers that would never have become clinically apparent. The recently published extended follow-up of the Canadian National Breast Screening Study is likely to provide reliable estimates of the extent of overdiagnosis. After 25 years of follow-up, it found that 106 of 484 screen-detected cancers (21.9\%) were overdiagnosed. ${ }^{3}$ This means that 106 of the 44,925 healthy women in the screening group were diagnosed with and treated for breast cancer unnecessarily, which resulted in needless surgical interventions, radiotherapy, chemotherapy, or some combination of these therapies. In addition, a Cochrane review of 10 trials involving more than 600,000 women 


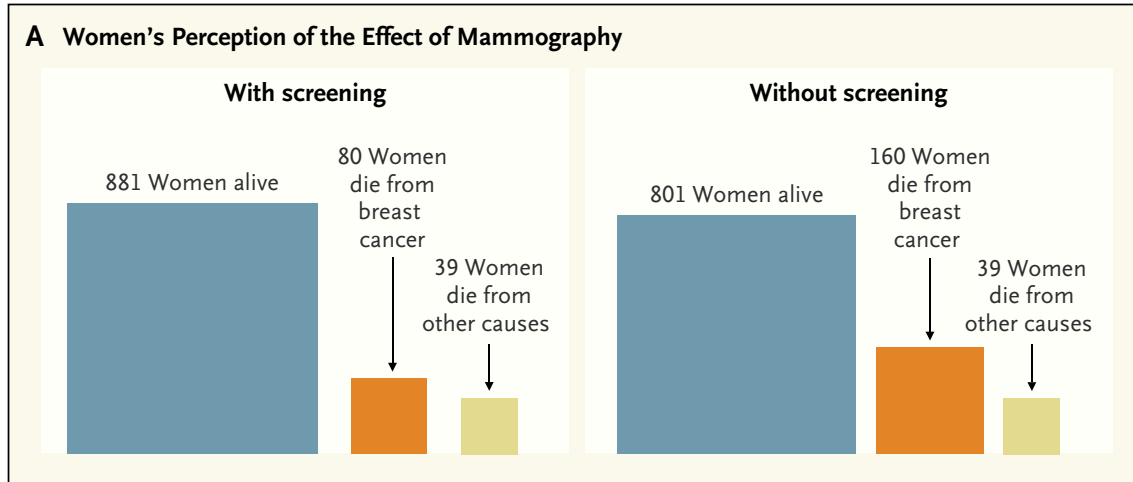

B Real Effect of Mammography

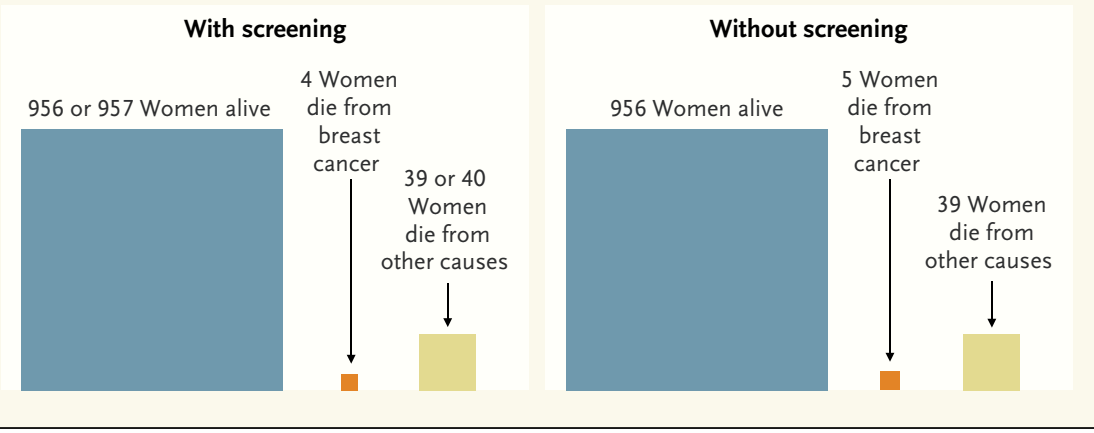

U.S. Women's Perceptions of the Effects of Mammography Screening on Breast-Cancer Mortality as Compared with the Actual Effects.

Panel A shows the views of 50-year-old women in the United States regarding the effect of mammography every 2 years on the 10-year risk of death from breast cancer (at left), as compared with no screening (at right). The areas of the squares are proportional to the numbers of women per 1000 who would be alive (blue), die from breast cancer (orange), or die from other causes (yellow). The numbers were calculated from women's perceived relative and absolute risk reductions for breast-cancer deaths (Domenighetti et al. ${ }^{4}$ ) and U.S. mortality statistics for 2008 from the Centers for Disease Control and Prevention. Panel B shows the actual effect of mammography screening on breast-cancer deaths, with numbers calculated from breast-cancer mortality data for 2008 from the National Cancer Institute and U.S. mortality statistics for 2008, assuming a relative risk reduction of $20 \%$ for breast-cancer mortality in women invited to undergo screening (Independent U.K. Panel${ }^{2}$ ).

showed there was no evidence suggesting an effect of mammography screening on overall mortality. ${ }^{1}$ In the best case, the small reduction in breast-cancer deaths was attenuated by deaths from other causes. In the worst case, the reduction was canceled out by deaths caused by coexisting conditions or by the harms of screening and associated overtreatment. Did the available evidence, taken together, indicate that mammography screening indeed benefits women?

Third, we were disconcerted by the pronounced discrepancy between women's perceptions of the benefits of mammography screening and the benefits to be expected in reality. The figure shows the numbers of 50-year-old women be alive, to die from breast cancer, or to die from other causes if they are invited to undergo regular mammography every 2 years over a 10-year period, as compared with women who do not undergo mammography. The numbers in Panel A are derived from a survey about U.S. women's perceptions, ${ }^{4}$ in which 717 of 1003 women (71.5\%) said they believed that mammography reduced the risk of breast-cancer deaths by at least in the United States expected to half, and 723 women (72.1\%) thought that at least 80 deaths would be prevented per 1000 women who were invited for screening. The numbers in Panel B reflect the most likely scenarios according to available trials ${ }^{1-3}$ : a relative risk reduction of $20 \%$ and prevention of 1 breast-cancer death. The data for Switzerland, reported in the same study, show similarly overly optimistic expectations. How can women make an informed decision if they overestimate the benefit of mammography so grossly?

The Swiss Medical Board's report was made public on February 2, 2014 (www.medical-board.ch). It acknowledged that systematic mammography screening might prevent about one death attributed to breast cancer for every 1000 women screened, even though there was no evidence to suggest that overall mortality was affected. At the same time, it emphasized the harm - in particular, false positive test results and the risk of overdiagnosis. For every breast-cancer death prevented in U.S. women over a 10-year course of annual screening beginning at 50 years of age, 490 to 670 women are likely to have a false positive mammogram with repeat examination; 70 to 100 , an unnecessary biopsy; and 3 to 14, an overdiagnosed breast cancer that would never have become clinically apparent. ${ }^{5}$ The board therefore recommended that no new systematic mammography screening programs be introduced and that a time limit be placed on existing programs. In addition, it stipulated that the quality of all forms of mammography screening should be evaluated and that clear and balanced information should be provided to women regarding the benefits and harms of screening.

The report caused an uproar 
and was emphatically rejected by a number of Swiss cancer experts and organizations, some of which called the conclusions "unethical." One of the main arguments used against it was that it contradicted the global consensus of leading experts in the field - a criticism that made us appreciate our unprejudiced perspective resulting from our lack of exposure to past consensus-building efforts by specialists in breastcancer screening. Another argument was that the report unsettled women, but we wonder how to avoid unsettling women, given the available evidence.

The Swiss Medical Board is nongovernmental, and its recommendations are not legally binding. Therefore, it is unclear whether the report will have any effect on the policies in our country. Although Switzerland is a small country, there are notable differ-

cil An audio interview with Dr. Mette Kalager about the Swiss Medical Board recommendation is available at NEJM.org ences among regions, with the French- and Italianspeaking cantons being much more in favor of screening programs than the German-speaking cantons - a finding suggesting that cultural factors need to be taken into account. Eleven of the 26 Swiss cantons have systematic mammography screening programs for women 50 years of age or older; two of these programs were introduced only last year. One German-speaking canton, Uri, is reconsidering its decision to start a mammography screening program in light of the board's recommendations. Participation in existing programs ranges from 30 to $60 \%$ - variation that can be partially explained by the coexistence of opportunistic screening offered by physicians in private practice. At least three quarters of all Swiss women 50 years of age or older have had a mammogram at least once in their life. Health insurers are required to cover mammography as part of systematic screening programs or within the framework of diagnostic workups of potential breast disease.

It is easy to promote mammography screening if the majority of women believe that it prevents or reduces the risk of getting breast cancer and saves many lives through early detection of aggressive tumors. ${ }^{4}$ We would be in favor of mammography screening if these beliefs were valid. Unfortunately, they are not, and we believe that women need to be told so. From an ethical perspective, a public health program that does not clearly produce more benefits than harms is hard to justify. Providing clear, unbiased information, promoting appropriate care, and preventing overdiagnosis and overtreatment would be a better choice.

The views expressed in this article are those of the authors and do not necessarily reflect those of all members of the expert panel of the Swiss Medical Board.

Disclosure forms provided by the authors are available with the full text of this article at NEJM.org.

From the Institute of Biomedical Ethics, University of Zurich, Zurich (N.B.-A.), and the Institute of Social and Preventive Medicine and Clinical Trials Unit Bern, Department of Clinical Research, University of Bern, Bern (P.J.) - both in Switzerland; and the Division of Medical Ethics, Department of Global Health and Social Medicine, Harvard Medical School, Boston (N.B.-A.). Dr. BillerAndorno is a member of the expert panel of the Swiss Medical Board; Dr. Jüni was a member of the panel until August 30, 2013.

This article was published on April 16, 2014, and updated on May 1, 2014, at NEJM.org.

1. Gøtzsche PC, Jørgensen KJ. Screening for breast cancer with mammography. Cochrane Database Syst Rev 2013;6:CD001877.

2. Independent UK Panel on Breast Cancer Screening. The benefits and harms of breast cancer screening: an independent review. Lancet 2012;380:1778-86.

3. Miller AB, Wall C, Baines CJ, Sun P, To T, Narod SA. Twenty five year follow-up for breast cancer incidence and mortality of the Canadian National Breast Screening Study: randomised screening trial. BMJ 2014;348: g366.

4. Domenighetti G, D'Avanzo B, Egger M, et al. Women's perception of the benefits of mammography screening: population-based survey in four countries. Int J Epidemiol 2003;32:816-21.

5. Welch HG, Passow HJ. Quantifying the benefits and harms of screening mammography. JAMA Intern Med 2014;174:448-54.

DOI: 10.1056/NEJMp1401875

Copyright @ 2014 Massachusetts Medical Society.

\section{The Legality of Delaying Key Elements of the ACA}

Nicholas Bagley, J.D.

Tnder the Affordable Care Act (ACA), the employer mandate - the requirement that most employers offer health insurance to their workers or pay a tax penalty - was scheduled to go into effect on January 1, 2014. Last summer, however, the Obama administration announced that it was delaying the mandate for a year. The administration has now extended the delay for midsize firms until 2016.

The latest delay has spurred another round of accusations from critics of health care reform that the Obama administration has acted unlawfully in implementing the ACA. Similar accusations followed the announcement of a 1-year delay for some insurers of the ACA caps on outof-pocket costs, as well as the decision to allow people to keep 